\title{
Standard Model $H \rightarrow \gamma \gamma$ discovery potential with ATLAS
}

\section{Yaquan FANG*}

On Behalf Of The ATLAS Collaboration

University of Wisconsin, Madison

E-mail: yaquan.fang@cern.ch

This contribution summarizes the discovery potential of the Standard Model Higgs boson using the $H \rightarrow \gamma \gamma$ decay with the ATLAS detector. The relevant detector performance aspects of photon reconstruction, photon identification and trigger issues are discussed. The potential of inclusive $H \rightarrow \gamma \gamma$ as well as Higgs boson searches in association with one or two hard jets are studied. The discovery potential is finally assessed using an unbinned multivariate maximum-likelihood fit with an expected integrated luminosity of $\approx 10 \mathrm{fb}^{-1}$.

Physics at LHC 2008

29 September - October 4, 2008

Split, Croatia

${ }^{*}$ Speaker. 


\section{Introduction}

In the light Higgs mass range $115<m_{H}<140 \mathrm{GeV}, H \rightarrow \gamma \gamma$ is one of the most promising channels. Excellent photon energy reconstruction and angular resolution are required to observe a narrow diphoton mass peak above the $\gamma \gamma$ QCD invariant mass spectrum. Furthermore, an effective photon identification is necessary to reject the huge rate of QCD jets and $\gamma$-jets. This channel has been studied for a long time [1]. The inclusive study is revisited with the most up-to-date detector geometry and software. The impact of higher order QCD and EW corrections is also considered in this study (see Ref. [2]). The analysis of Higgs decaying into two photons in association with one or two hard jets is re-evaluated with a more complete description of the detector simulation. The analyses corresponding to associated processes $W H, Z H$ and $t \bar{t}$ are also updated and documented in Ref [2]. In addition to the invariant mass of the photon pair, other kinematics and different topological properties are also taken into account to enhance the sensitivity of the analysis. All the aspects are incorporated by means of a simultaneously unbinned maximum-likelihood fit.

\section{Experimental aspects of the analysis}

\subsection{Trigger items}

Two trigger menus $2 \mathrm{~g} 17 \mathrm{i}$ and $\mathrm{g} 55$ are expected for $H \rightarrow \gamma \gamma$ analysis. The former selects events with two isolated photons with $p_{T}>20 \mathrm{GeV}$. At least one photon with $p_{T}>60 \mathrm{GeV}$ is required for selected events in the g55 trigger. In this study, $2 \mathrm{~g} 17 \mathrm{i}$ is used to avoid the biases in the diphoton mass reconstruction. The signal's trigger efficiency, normalized to inclusive analysis cuts, is $94 \%$.

\subsection{Photon identification and jet rejection}

Powerful photon identification is crucial for a search in the $H \rightarrow \gamma \gamma$ channel. A single jet rejection with a factor of 5000 is required while keeping the high transverse momentum photons $\left(p_{T}>25 \mathrm{GeV}\right)$ with an efficiency of $\sim 80 \%$. Dedicated cuts [2] were applied on shower shape variables based on EM calorimeter depositions and track isolation. The rejection for an inclusive jet with track isolation cut is $8160 \pm 250(5070 \pm 120$ without track isolation cut). The rejection against gluon initiated jets is a factor of 10 higher than that of quark initiated jets, mostly due to the different fragmentation characteristics. The average EM calorimeter cut efficiency for signal sample has been found to be $\sim 83 \%$, even in presence of pile-up. The track isolation cut efficiency is $\sim 98 \%$.

\subsection{Conversion reconstruction}

Converted photons showering upstream can result in the energy resolution worse than that of the unconverted photons. In addition, their energy deposition in the calorimeter is geometrically wider in the $\phi$ direction due to the magnetic field in the Inner Detector. Nevertheless, the track of the converted $e^{+} / e^{-}$can help to improve the determination of the primary vertex. Photon conversion needs to be taken into account carefully since around $\sim 57 \%$ of selected events have at least one converted photon. A vertexing algorithm using a reconstructed particle track has been developed to associate an EM cluster (converted photon candidate) with two or one tracks, namely double track 
conversion or single track conversion. The tagging efficiency as a function of conversion is shown in Figure 1.

\subsection{Primary vertex reconstruction}

Precise measurement of $z$ vertex of the two photons can help to determine the $\eta$ of the photons, thereby enhancing the mass resolution of the reconstructed Higgs. To do this, linear fits are implemented in the $(R, z)$ plane through the cluster barycenters detected in the presampler and in the first and second samplings of the EM calorimeter. The intercept of the lines with the beam axis provides the hard scattering vertex of the photons. Combining them and the nominal interaction vertex at $z_{0}=0$ yields an estimation of $z$ vertex of Higgs boson based on measurements in the calorimeter, $Z_{H}^{\text {calo }}$. In the case of conversion, the Higgs boson vertex position accuracy can be improved by including the pointing of the conversion track. By adding the reconstructed event vertex to the linear fit, the best Higgs boson position accuracy is achieved with a Gaussian width of 0.07 $\mathrm{mm}$ as Figure 2 shows. The discrimination of the hard-scattering vertex from those due to pile-up is done using a likelihood method.

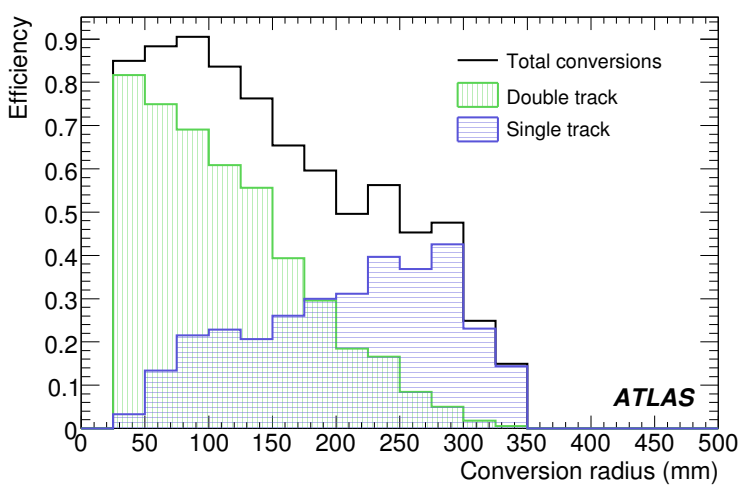

Figure 1: Efficiency of single and double track conversion reconstruction as a function of conversion radius.

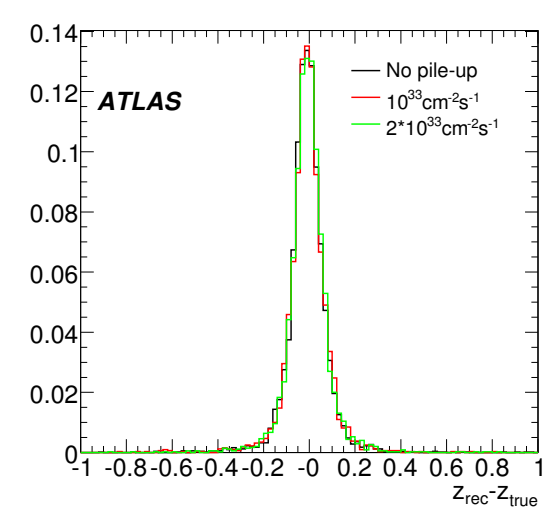

Figure 2: Calorimetric pointing with the reconstructed primary vertex.

\subsection{Invariant mass reconstruction}

Unconverted photons and converted photons are reconstructed from clusters of different size. The energy of the photons in the EM calorimeter has been reconstructed and calibrated considering a series of effects. The invariant mass of the diphotons has been reconstructed after the trigger and inclusive cuts. Figure 3 presents the invariant mass distributions for diphotons from Higgs boson decays with $m_{H}=120 \mathrm{GeV}$. The mass resolution has been determined from an asymmetric Gaussian fit $([-2 \sigma,+3 \sigma])$ to the invariant mass peak. The relative mass resolution $\sigma_{m} / m$ is close to $1.2 \%$ degrading by a few percent when pile-up is added.

\section{Event Selection}

\subsection{Inclusive analysis}

The signature for the inclusive analysis is simply two energetic photons. We require that two 


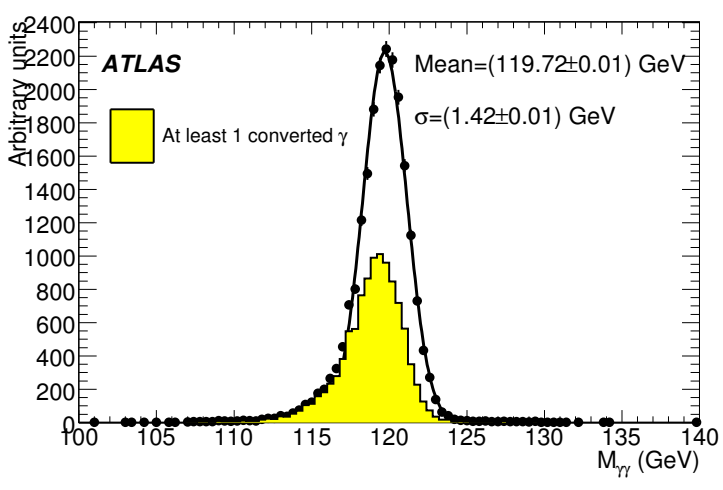

Figure 3: Invariant mass distributions for diphotons from Higgs boson decays with $m_{H}=120$ $\mathrm{GeV}$. The shaded histogram corresponds to events with at least one converted photon.

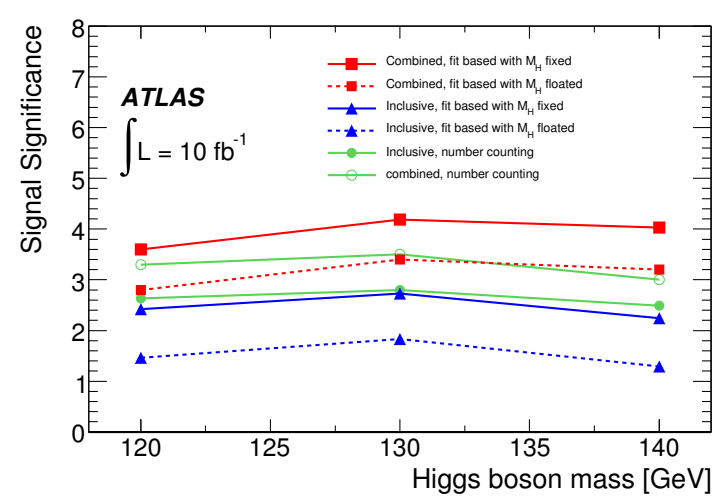

Figure 4: Expected signal significance for a Higgs boson using the $H \rightarrow \gamma \gamma$ decay for $10 \mathrm{fb}^{-1}$ of integrated luminosity as a function of the mass (see text for more explanation).

photon candidates in the central detector region, defined as $|\eta|<2.37$, excluding the crack region between barrel and end-cap calorimeters, $1.37<|\eta|<1.52$. At this level it is required that the events pass the trigger selection. Transverse momentum cuts of 40 and $25 \mathrm{GeV}$ are applied on the leading and sub-leading photon candidates, respectively.

\subsection{Higgs boson plus one jet and Higgs boson plus two jets analysis}

Photonic cuts for the Higgs boson plus one jet analysis are similar to those of the inclusive case, except that the $p_{T}$ cut on leading photon is $45 \mathrm{GeV}$ instead of $40 \mathrm{GeV}$. In addition, we require at least one hadronic jet with $p_{T}>20 \mathrm{GeV}$ in $|\eta|<5$. The invariant mass of the diphotons and the leading jet $\left(m_{\gamma \gamma j}\right)$ is used as a discriminator to improve the signal-to-background ratio. The cut on it is $m_{\gamma \gamma j}>350 \mathrm{GeV}$.

The transverse momentum cut on the leading photon for the Higgs boson associated with two jets is $5 \mathrm{GeV}$ harder than that for Higgs boson plus one jet analysis. In addition, at least two hadronic jets are required in $|\eta|<5$ with $p_{T}>40,20 \mathrm{GeV}$ for the leading and sub-leading jet, respectively. These two tagging jets are required to be in opposite hemispheres and pseudo-rapidity gap between them should be larger than 3.6. Photons are required to have pseudorapidity between those of the tagging jets. The invariant mass of the tagging jets $m_{j j}$ is required to be greater than $500 \mathrm{GeV}$. Finally, we veto any event with a third jet which has $p_{T}>20 \mathrm{GeV}$ and $|\eta|<3.2$.

\section{ATLAS Higgs discovery potential in the $\gamma \gamma$ channel}

This section reports the discovery potential of a Higgs boson in the mass range $120<m_{H}<$ $140 \mathrm{GeV}$. In inclusive analysis case, the expected signal significances using event counting for 10 $\mathrm{fb}^{-1}$ of integrated luminosity are 2.6, 2.8 and 2.5 with respect to Higgs boson masses of 120, 130 and $140 \mathrm{GeV}$. Since the sensitivity for the inclusive, $\mathrm{H}+1 \mathrm{jet}$ and $\mathrm{H}+2 \mathrm{jets}$ selections are different, a combination of them taking into account the event overlap among the three analyses, can enhance the significance to $3.3(120 \mathrm{GeV}), 3.5(130 \mathrm{GeV}), 3.0(140 \mathrm{GeV})$. 
In parallel, the significances are computed using the maximum-likelihood fit formalism. With respect to the cut analyses, the fit procedure takes advantage of further discrimination information from the kinematic and topological properties of $H \rightarrow \gamma \gamma$ decays. In addition to the diphoton mass, $m_{\gamma \gamma}$, the transverse momentum of the Higgs boson, $P_{T, H}$, and the magnitude of the photon decay angle in the Higgs boson rest frame with respect to the Higgs laboratory flight direction, $\left|\cos \left(\theta^{*}\right)\right|$, are included. Three photon pseudorapidity regions according to the resolution of $m_{\gamma \gamma}$ and three jet categories as well as photon conversion are introduced to classify events during the fit processes. The significances are extrapolated in both cases of fixed and floating Higgs mass. Figure 4 displays a summary of the expected signal significance for the inclusive and final combined analysis for 10 $\mathrm{fb}^{-1}$ of integrated luminosity as a function of the Higgs boson mass. The solid and hollow circles correspond to the sensitivity of the inclusive and combined analysis using event counting. The solid triangles linked with solid and dashed lines correspond to the sensitivity of the inclusive analysis by means of one variable $\left(m_{H}\right)$ fits, with a fixed and floating Higgs boson mass, respectively. The solid squares linked with solid and dashed lines correspond to the values of significance using three kinematics variables fits and all event classifications, with a fixed and floating Higgs boson mass, respectively. The solid squares linked with solid and dashed lines correspond to the maximum sensitivity that can be attained with a combined analysis, which enhance the significance by at least $40 \%$ with respect to the inclusive case.

\section{Conclusion}

The feasibility of the search for the Standard Model Higgs boson in the $H \rightarrow \gamma \gamma$ decay with the ATLAS detector at the LHC has been evaluated. The main experimental aspects of the analysis have been evaluated using the updated full detector simulation. The $5 \sigma$ signal significance are expected to be accessed with integrated luminosity $20-30 \mathrm{fb}^{-1}$.

\section{References}

[1] L. Fayard and G. Unal, ATL-PHYS-92-001 (1992); The ATLAS Collaboration, CERN/LHCC/99-14/15 (1999); M. Bettinelli, et al., ATL-PHYS-PUB-2007-013 (2007); S. Abdullin et al., Phys. Lett.B431 (1998) 410; D.L. Rainwater and D. Zeppenfeld, JHEP 9712 (1997) 005; D.L. Rainwater, PhD thesis, hep-ph/9908378 (1999); S. Zmushko, ATL-PHYS-2002-020 (2002); K.Cranmer et al., ATL-PHYS-2003-036 (2003), hep-ph/0401088; M. Escalier, CERN-THESIS-2005-023;

[2] The ATLAS Collaboration, CERN-OPEN-2008-020; 